\title{
Informational Neuro-Connections of the Brain with the Body Supporting the Informational Model of Consciousness
}

\author{
Florin Gaiseanu* \\ Science of Information and Technology Bucharest (Romania) and Barcelona, Spain
}

*Corresponding author: Florin Gaiseanu, PhD, Principal Senior Researcher (Professor), Science of Information and Technology Bucharest (Romania) and Barcelona, Spain.

Received Date: June 10, 2019

Published Date: June 12, 2019

\section{Abstract}

Introduction: The objective of this investigation is to analyse the informational circuits of the brain connections with the body from neurologic and neuroscience point of view, on the basis of the concepts of information promoted by the Informational Model of Consciousness.

Analysis: Distinguishing between the virtual and matter-related information promoted by the Informational Model of Consciousness, the main specific features of consciousness are analyzed from the informational perspective, showing that the informational architecture of consciousness consists in seven groups of specific activities, defined as cognitive centres, each of them with specific distinct tasks, but correlated each other: centre of acquisition and storing of information (memory), centre of decision and command (decision), centre of the emotional states (emotions), centre of the body maintenance (power and health), centre of the genetic elaboration/transmission (reproduction) and the info-genetic generator, inherited from the parents (predispositions, talents and skills). A special centre, dedicated to the connectivity with some extra-power properties of the mind is also introduced, assuring an intimate supra-sensitive detection of the world to explain the associated phenomena of the near-death experiences.

Result: The activity of all these centres should be supported neurologically by the brain neuro-connectivity to the body and to external and internal info-signals. On the basis of the informational analysis, the neuro-connections of the brain regions associated to the main characteristics of the cognitive centres are highlighted, showing the anatomic and neuro-functional relation between the distinct components of the brain and the specific operating body regions. These connections describe in terms of information the brain-body neuro-activities as informational specific circuits, composed by the info-operational subsystems managed by the brain, and sensors, transducer and execution elements.

Conclusion: The components and connections mind-body stipulated by the Informational Model of Consciousness are supported by the neurologic/neuroscience evidences.

Keywords: Brain; Informational operations; Cognitive centers; Informational characteristics; Brain-body neuro-connections; Informational circuits

\section{Introduction}

When the brain activities and properties are discussed within the scientific community and during our daily activities, their informational character it is often invoked. However, as the concept of information is today very commonly used in various areas of human activities, especially when we refer to the large range of information channels, like digital telephony, internet, television and computers, little attention is paid to the real significance of this concept when we refer to the informational characteristics of the brain. We receive information from our environment, allowing to connect to the reality under a permanent dynamic process, and we operate this information to find a suitable/optimal way for adapting response. We also communicate with our body, controlling the spontaneous internal impulses or accessing to its internal informational signals in order to comply with its needs and requirements. This seems already to be a regular working mode and no attention is paid on the deep significance of such a communication both with external and internal reality, or on what really means information and the associate circuits inside of our body [1]. 
Consciousness was and is a largely debated topic, because the nature of consciousness and the associated working mechanisms are still not understood. Although information is a largely accepted concept applicable to the mind operation, not real advances were reordered to explain on this basis the characteristics and properties of the mind. The mechanisms of the mind were recently approached from the perspective of a processor-like operating system [2], in order to discern the various levels of operating possibilities, from the quantum physics to understand the computational mode of brain operation [3], or from informational perspective to describe the integrative functions of the brain [4]. However, only recently an Informational Model of Consciousness taking into account the specific properties of consciousness from the informational point of view was reported $[1,5]$.

Starting from an analysis of the information concepts promoted by the Informational Model of Consciousness, the neuroconnections of the brain with the body and the informational neurocircuits as a function of various types of information managed by the brain are revealed, supporting the informational model from the neuroscience and neurological perspective.

\section{Analysis}

\section{Analysis of the informational components of consciousness and their operability}

In Figure 1 the components of consciousness as promoted by the Informational Model of Consciousness (IMC) [1,5] are represented showing a seven-centres type architecture. These centres reflects the main distinctive functions of the brain from the informational point of view. However, to understand consciousness from the informational perspective according to such functions, an analysis of the distinctive concepts of information should be performed. The mind operates with virtual information. When we receive information, specifically the visual one, this is represented on our virtual "screen", allowing a spontaneous and continuous visual detection of the surrounding reality. When we activate a memory recorded information, this is also "viewed" by the internal "eye" of the mind on the internal virtual "screen". Therefore, the operated information by the mind is a virtual information.

Within the range of mentally operated information, we have to distinguish also between two main categories. One category is the "stand-by" information, accumulated in memory, which is still not active, like in a computer memory. From IMC point of view, the informational operator of the mind is the thought, which allows to activate a certain type of information, bringing it on the virtual screen, either from external, internal or memory domain. Therefore, we have to distinguish this category as an active, dynamic information, which should be attributed to a distinct type of activity, associated to a distinct area of the brain. Within IMC, this distinction is reflected by two defined centres: I-know (abbreviated by Ik in Figure 1), which consists in the accumulated info-luggage during the life, and the operative informational centre defined as I-want (Iw in Figure 1), which elaborates the decision, on the basis of the info-detected or accumulated data. The decisional process is based on information and the decisional criteria, which allows the selection between accepted (YES) or not accepted (NO) information [6], like in the computer systems [7], where the information is operated by a binary YES/NO procedure (Figure 1).

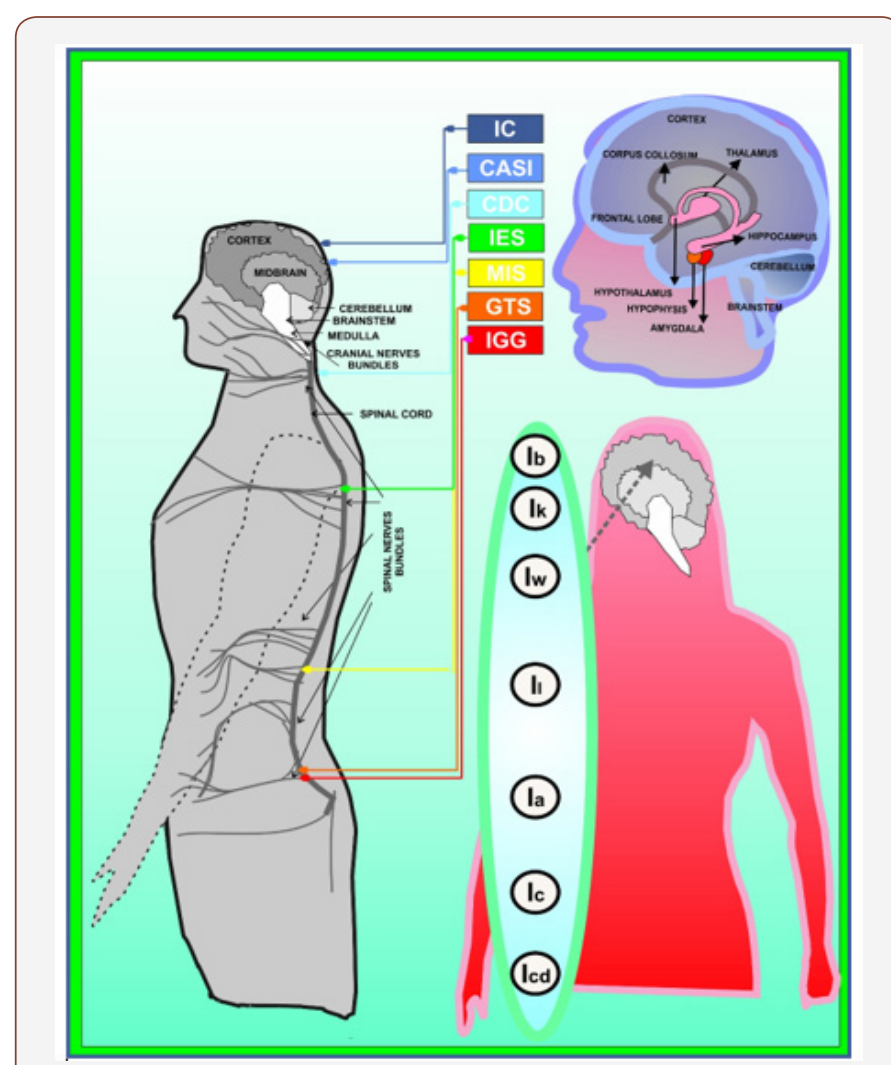

Figure 1: Consciousness components (right side) consisting in seven cognitive centers according to IMC, and the brain-body informational neuro-connections (left and upper side).

In the science of information and technology, the concept of information is sharply defined by functions of probability in a binary system $[8,1]$, the quantity of information in a communication system being calculated as a difference between the entropies of two states of a system. The entropy is a measure of uncertainty, while information is a measure of certainty [9]. In physics, the entropy is a measure of "freedom" degrees of a system, which often are referred as to a disorder degree. On contrary, information is assimilated to certainty and ordered system.

Emotions are generated as a reaction to the virtual information and are detected in the conscious mind by two main emotional categories: impulsive/spontaneous emo-reactions and emotional states (emo-states) [6]. Therefore, the emotions are also informational signals and should be included in a specific distinct category. Within the IMC, these emo-signals were attributed to the info-emotional centre defined as I-love (Il in Figure 1). This suggestive name comes from the large spectrum of emotions associated to love. I-love manages automatically the emo-signals, as an immediate reaction to the external stimuli, or info-induced emotions associated with the remembrances, as info-memorized information. From this point of view, this centre is an intermediary operating centre between the automatically triggered and voluntarily managed emotional information. 
A special category of informational activity is dedicated to the management of digestive and breathing processes, necessary to maintain the power and the functional material and energetic support of the organism. This category of informational activity is fully automatic, the internal associated signals only advertising on the body absorbing/desorbing necessities (hanger, thirst) or on momentary state of the body. However, at the global level, this activity is detected in the conscious mind as an average power/ health/vitality status from the own self perspective. Therefore, this centre was defined as I-am centre (Ia in Figure 1), because it is dedicated to the body material and energetic maintenance.

From the informational point of view, the genetic information is a matter-related information, because the information itself is contained/integrated into the cell. The characteristic information is carried by the genes. Although the concept of matter-related information could seem to be no usual, this form of expression points out the relation between information and its matter support. Within the mater-related informational systems, the information is intrinsic, contained into the intimate structure of the material system, formed by multi-particles components. All the material structures are composed by atoms and sub-particles. These components are unified by specific forces and can be characterized by entropy and information. When the degree of atomic order is high, like for instance in the case of silicon semiconductor materials, the basic material for the fabrication of the intelligent processors, microelectronic structures and integrated microsystems [10-13], the entropy is low and such structures contain a high quantity of incorporated information. If one atoms abandons its particular site, defined by the physical and mathematical laws of the lattice (marked by a minimal potential), then the entropy of the system increases and implicitly the disorder (or freedom) degree, by emission of information. Therefore, these were specifically defined as processes of embodiment/disembodiment of information. An integrated atom into a specific site incorporates (embodies) information, as related by all its valence bonds with the neighbourhood atoms in the structural lattice, but when these inter-related bindings are broken, the system turns into a new state, with higher entropy, because an incorporated information is lost by this info-disembodiment process.

The genetic information is similarly incorporated (embodied) into the genetic components of the cells, as matter-related information, so when some specific conditions are fulfilled, this genetic information is generated by an info - disembodiment process. The epigenetic processes, allowing to transfer to the offspring the info-acquired traits during the life, follow the same type of informational mechanisms [14]. It is therefore justified to define a specific centre managing the genetic information, suggestively called I-create (Ic in Figure 1). This centre is responsible for the genetic support of procreation, and with the formation of the new generation, being closely related to the centre I-love.

As the new human entity is a product of the genetic information from parents, it is also justified therefore to define an informational centre called I-created (Icd in Figure 1), which is reflected, besides through the main characteristics of the species, by the transmitted traits, predispositions, talents and skills.

Therefore, at the global level, the personal usual characteristics of I, defined as I-usual (Iu), could be expressed as a sum of all contributing centres activities as follows:

$$
\mathrm{Iu}=\mathrm{Ik}+\mathrm{Iw}+\mathrm{Il}+\mathrm{Ia}+\mathrm{Ic}+\mathrm{Icd}
$$

However, a special category of sensorial capacity of the mind should be attributed to the detection of the near-death (NDEs) $[15,16]$ and religious and mystic (RMEs) [14] experiences, besides other extra-sensorial phenomena like premonition [17]. These properties are based on the mind power, which is able to detect extra-corporal experiences during NDEs. Such states are experienced during the clinically proved cessation of the brain and hearth activities and are typically the same both for normal and blind people, who never benefited of the normal view [18]. Therefore, to complete the range of the distinct components of consciousness, we have to define the centre suggestively called I-believe (Ib in Figure 1), detected in the mind by peace, safety and confidence $[7,19-21]$ like during the practice of RMEs or meditative states $[14,22]$.

Therefore, the personal I could be generally described by the relation:

$\mathrm{I}=\mathrm{Iu}+\mathrm{Ib}=\mathrm{Ik}+\mathrm{Iw}+\mathrm{Il}+\mathrm{Ia}+\mathrm{Ic}+\mathrm{Icd}+\mathrm{Ib}$

This relation shows that each individual is a distinct entity, not only as the matter structure is concerned, but also from informational point of view, according to the personal features inherited from the parents and the habits acquired during the life.

\section{Result}

\section{Informational neuro-connections of the brain with the body elements}

A question which arises from the above analysis is what the corresponding areas of the brain are supporting the described functions and what are the corresponding circuit connections with the body from the neurological and neuroscience point of view. In Figure 1 (left and upper side) are represented both the corresponding areas of the brain (upper side) and the neuroconnecting circuits with the body (left side).

The informational subsystems corresponding to the components of consciousness described above could be defined as [1]: CASI, which is the Centre of Acquisition and Storing of Information (perception and memory), CDC, which is the Centre of Decision and Command (decision making and command transmission, especially to the motor components of the body - muscles), IES, which is the Info-Emotional System (emotions), MIS - the Maintenance Informational System (absorption/desorption and distribution processes), GTS - the Genetic Transmission System, IGG - InfoGenetic Generator and IC - Info-Connection, which corresponds to the super-sensitive phenomena. 
According to the associated characteristics of each subsystem defined above and the associated cognitive centre, the regions of the brain where the specific activities are operated could be identified $[2,23]$ as presented below. CASI is a sum of the specific areas of the brain associated with the short and long-term memory, composed by the prefrontal cortex (short-term memory, 1 min. duration), hippocampus - long-term memory, cerebellum, (learned behaviours and skills), thalamus - a relay for sensory impulses, cerebral cortex interpreting the main sensorial signals (touch, vision, hearing). CASI is connected to the external and internal sensors, which transmit to it the input information. CDC is a sum of the associated areas responsible for the main dynamic abilities of the mind like thought, creativity, communication, intelligence, motivation, judgement, planning, voluntary control of muscles, operated especially by the cerebral hemispheres, frontal and prefrontal lobes of the cerebrum. Cerebellum has also a helping role in the muscles coordination, including learning and storing motor skills. The left hemisphere is typically responsible for language and speech, comprehension, arithmetic, and writing, while the right hemisphere is dedicated especially to the creativity, spatial ability, artistic and musical skills. The decisional information is communicated by attitude, which provides to the body and to the external environment a decisional information, as an information output. The motor decisions are addressed to the execution elements, mainly to the muscles.

IES, as a sum of various components of the brain, is managed by the limbic system, and represented by thalamus, hypothalamus, hippocampus, midbrain and amygdala. MIS is composed by the brain stem area, as a relay between the cerebrum and cerebellum to the spinal cord, controlling many automatic functions such as breathing, heart rate, body temperature, wake and sleep cycles and digestion, while the hypothalamus manages and integrates the activities of the autonomic nervous system. Medulla - the last part of the brain stem controls the vital autonomic reflex functions like cardiac activity - the rate and force of heartbeat, vasomotor regulation, variation of diameter of the blood vessels, the blood distribution to specific organs, the blood pressure and the respiratory rate and depth of breathing.

GTS is correlated mainly with hypophysis and hypothalamus, responsible for the sexual activity. As it was commented above, this system assures the output matter-related (genetic) information of the body, including the species characteristics and the acquired traits. IGG manages the development of the body according to the age by means of hypophysis and hypothalamus, regulating the body growth and its development, metabolism and even the aging [24]. Basal ganglia seems to have also a role in the personality features. This system is based on the genetic input information inherited from parents.

IC pole area could be assimilated with the anterior cingulate cortex, as it was recently reported [25]. The intimate processes in this range of activity, explained by the implication of the quantum physics in biologic organisms [26], particularly in NDEs [15-17] and in RMEs [22], are also compatible with the meditative and palliative therapy processes $[19,27,28]$. The components described above represents the info-operational systems of the body [1]. However, to manage the body by means of information, these systems should be connected to sensors - the sensitive elements of the body transmitting information from the internal and external sources, and to transducers and motor-type execution elements, responsible to convert the info-signals emitted by the brain into the chemical or physical action. The informational circuits connecting the brain with such elements are mainly represented by the nervous system [23]. In Figure 1 (left and upper side) are represented the neuroconnections between the brain and each corresponding region of the body by means of the spinal cord and by the nervous bundles, spreading the information to the specific executive organs.

These circuits are represented in Figure 1 by colored lines. The violet and indigo lines indicate the neuro-connection with IC pole and CASI, with the headquarter and interpreting zones in the brain itself. The blue line connects CDC with the main execution element expressing the attitude-the communication transducer - which is the vocal system. The green line neuro-connects the limbic emotional system with the heart, which is a main sensitive element of emotions. However, as far as the functions of the heart are concerned, the automatic/rhythmic processes managed by the brain for the body maintenance (integrated in MIS), should not be confused with the dynamic activity induced by emotions as overlapping signals, necessary to manage the blood distribution, the vessel dynamics and blood pressure, according to the momentary regulating requirements.

The yellow line represents the neuro-connection of MIS with the nervous zone mainly responsible for the metabolic digestive absorption/desorption processes. This zone is also closely related to the hearth and lung activities for the distribution of the nutritional elements by the blood and for the air absorption/ desorption activities respectively. The orange and red colored lines represents the neuro-connections with the reproduction system.

The above discussion reveals first of all that the neurological/ neuroscience evidences supports the Informational Model of Consciousness. On the other hand, this investigation shows that the human body is a bipolar structure, composed by information and matter. The informational system is managed by the brain and transmitted by means of the nervous system, as an informational distribution support. The received information acquired by this system can be incorporated into the cell by epigenetic overlapping processes, which can be transmitted to the offspring, through the genetic matter-related support, an expression of the maximum level of info-integration in matter, able for reproduction. On this way, the information has a primary role in the modelling of the body material components, the mind-body complex acting as a reactive/ adaptive info-material system.

\section{Conclusion}

The analysis of the distinct functions of the brain from the informational perspective was possible on the basis of the distinction between the virtual information, operated by mental processes, and 
the matter-related information, particularly genetic information, incorporated into mater, as an info-material support. It was defined the process of the embodiment/disembodiment of information, as a basic process describing the interaction of information with matter, which allows to understand the mechanisms of the info-integration into (or info-emission from) the matter-related components, from the perspective of the Informational Model of Consciousness. This analysis allowed to reveal the main and distinct categories of brain informational activities, grouped as follows: info-perception and memory, info-operability and decisional activity, info-emotional activity, automatic maintainability of the body, info-genetic transmission and info-genetic reception. A special category of informational activity was attributed to NDEs and RMEs.

To associate such operational activities with the anatomic components of the brain and the body, the specific areas and components of the brain, dedicated to each function were identified. The informational circuits neuro-connecting the brain with each corresponding region of the body by means of the spinal cord and the nervous bundles as distributers of the information toward the organs, which acts actually as info-transducers and execution elements, were also revealed. The sensorial elements are connected to CASI, assuring the reception of suitable information from internal and external sources, for optimal operational decision. The above investigation shows first of all that the neurologic/neuroscience evidences supports the Informational Model of Consciousness. On the other hand, it reveals that the human body is actually a bipolar structure, composed by information and matter, where the information has a primary role in the modelling of the body material components, the mind-body complex acting as a reactive/ adaptive info-material system as a function of the external dynamic conditions.

\section{Conflict of Interests}

No conflict of interests.

\section{Acknowledgment}

To my son Adrian for his special virtues and qualities and to his family. To my daughter Ana for her aptitudes and virtues and to her family. To all members of family who appreciate and love me.

In the memory of my exemplar and loved parents, the distinguished (Magna cum Laude) Professor Emanoil Gaiseanu and the distinguished (Emeritus) Professor Florica Gaiseanu, to my Brother Professor Constantin Gaiseanu and to All Members of my Family who were part of my life - Popescu, Plopeanu, Potlogeanu, Tutuleasa, Vujdea.

\section{References}

1. Gaiseanu F (2018) Information: from Philosophic to Physics Concepts for Informational Modeling of Consciousness, Philosophy Study 8(8): 368-382.

2. Baars BJ and Gage N (2013) Cognition, Brain and Consciousness, 2nd ed., USA, Academic Press (Elsevier Sequoia).

3. Hameroff S (1998) Quantum Computation in Brain Microtubules? The Pennrose-Hameroff. 'Orch OR' Model of Consciousness. Philos. Trans. Royal Society London Ser A Math Phys Sci.356: 1869-1898.
4. Tononi G (2008) Consciousness as Integrated Information: A Provisional Manifesto. Biol Bull 215: 216-242.

5. Gaiseanu F (2019) Destiny or Free Will Decision? A Life Overview from the Perspective of an Informational Modeling of Consciousness Part I: Information, Consciousness and Life Cycle. Gerontology \& Geriatric Studies 4(1): 1-7.

6. Gaiseanu F (2018) Destiny or Free Will Decision? A Life Overview from the Perspective of an Informational Modeling of Consciousness Part II: Attitude and Decision Criteria, Free Will and Destiny. Gerontology \& Geriatric Studies 4(1): 1-7.

7. Gaiseanu F (2016) Consciousness as Informational System of the Human Body. Consciousness and Life Physics, Cosmology and Astrophysics Journal 16(1): 14-25

8. Shannon C (1948) The Mathematical Theory of Communication. Bell Syst Tech J 27: 379-423.

9. Gaiseanu F (2019) Language Patterns and Cognitive-Sentient Reality: Certainty/Uncertainty in Cognitive-Sentient Exploration of Reality, Chapter in Media Models to Foster Collective Human Coherence in the PSYCHecology, Ed. Stephen Brock Schafer, USA, IGI Global.

10. Gaiseanu F, Sachelarie M, Sachelarie D and Esteve J (1997) Analytical Modeling of the Gold Diffusion Induced Modification of the Forward Current Density Through the p-n Junctions. Solid State Phenomena 3738: 525-530.

11. Gaiseanu F, Cobianu C, Dascalu D (1993) Dependence of the Chemical Etching Rate and Etching Time on the Post-Implanted Diffusion Depth: Application for Membrane Achievement. Journal of the Material Science Letters 12: 1652-1653.

12. Gaiseanu F (2013) Contributions to the Modelling and Simulation of the Atomic Transport Processes in Silicon and Polysilicon and Applications. PROCEEDINGS OF THE ROMANIAN ACADEMY, Series A 4(4): 376-384.

13. Gaiseanu F (2017) Modeling and Simulation of the Impurity Diffusion and Related Phenomena in Silicon and Polysilicon Systems in Microfabrication and Micromachining Technologies. Annals of the Academy of Romanian Scientists, Series on Science and Technology of Information 10(1): 41-78.

14. Gaiseanu F (2019) The Informational Model of Consciousness: Mechanisms of Embodiment/Disembodiment of Information. Neuro Quantology 17(4): 1-17.

15. Gaiseanu F (2017) Quantum-Assisted Process of Disembody Under Near-Death Conditions: An Informational-Field Support Model." NeuroQuantology 15(1): 4-9.

16. Gaiseanu F (2018) Near-Death Experiences and Immortality from the Perspective of an Informational Modeling of Consciousness. Gerontology \& Geriatric Studies 2(3): 1-4.

17. Gaiseanu F (2017) An Information Based Model of Consciousness Fully Explaining the Mind Normal/Paranormal Properties. NeuroQuantology 15(2): 132-140.

18. Meijer D (2013) Immortality: Myth or Becoming Reality? On the Conservation of Information. Syntropy 3: 168-207.

19. Gaiseanu F and Graur A (2018) Cognitive Centers Related Attitude: Application for an Iterative Evaluation Method in Music-Based Therapy Process. Abstract Book of the Conference on Science of Consciousness (Co-Chair Prof. Stuart Hameroff, The University of Arizona-Center for Consciousness Studies, Tucson, Arizona). Consciousness and Education-Cognitive Development, Concurrent Session C27, 165: 2-7.

20. Gaiseanu F (2018) An Informational Modeling of Consciousness and Cognitive Centers. Proc. of the Human Project Brain (HPB) International Conference: Understanding Consciousness, a Scientific Quest for the 21st Century. 04-Models, simulation and emulation of consciousness, Barcelona: 21-22 June.

21. Gaiseanu F (2016) Informational Subsystems of the Consciousness. The Science of Consciousness, Abstract Book, TSC 2016 TUCSON. Ed. The University of Arizona Center for Consciousness Studies and University of Michigan Center for Consciousness Science, by Stuart Hameroff (The University of Arizona) and George Mashour (University of Michigan). 
22. Gaiseanu F (2019) Human/Humanity, Consciousness and Universe: Informational Relation, NeuroQuantology 17(5): 60-70.

23. Dispenza J (2007) Evolve Your Brain: The Science of Changing Your Mind, Health Communications, Inc. Deerfield Beach, Florida.

24. Zhang Y, Kim MS, Jia B, Yan J, Zuniga-Hertz PJ, and Han C (2017) Hypothalamic stem cells control ageing speed partly through exosomal miRNAs. Nature 548: 52-57.

25. Inzlicht M, Tullett A, Good M (2011) The need to believe: a neuroscience account of religion as a motivated process, Religion, Brain \& Behavior 1(3): 192-251.
26. Tarlaci S (2011) Quantum physics in living matter: from quantum biology to quantum neurobiology. NeuroQuantology 9 (4): 692-701.

27. Satsangi AK and Brugnoli MP (2018) Anxiety and psychosomatic symptoms in palliative care: from neuro-psychobiological response to stress, to symptoms' management with clinical hypnosis and meditative states. Annals of Palliative Medicine 7(1): 75-111.

28. Brugnoli MP, Pesce G, Pasin E, Basile MF, Tamburin S, Polati E (2015) The role of clinical hypnosis and self-hypnosis to relief pain and anxiety in severe chronic diseases in palliative care: a 2-year long-term follow-up of treatment in a nonrandomized clinical trial, Ann Palliat Med: 1-17. 